\title{
Monitoring vascular changes induced by photodynamic therapy using contrast-enhanced micro-computed tomography
}

\author{
Otilia C. Nasui ${ }^{1}$, Stuart K. Bisland ${ }^{2}$, Nancy L. Ford ${ }^{1,3^{*}}$ \\ ${ }^{1}$ Department of Physics, Ryerson University, Toronto, Canada \\ ${ }^{2}$ Division of Orthopaedic Surgery, McMaster University, Hamilton, Canada \\ ${ }^{3}$ Department of Oral Biological and Medical Sciences, University of British Columbia, Vancouver, Canada \\ Email: nlford@dentistry.ubc.ca
}

Received 3 January 2013; revised 3 February 2013; accepted 10 February 2013

\begin{abstract}
The aim of this study was to determine whether contrast-enhanced micro-computed tomography can be used for non-invasive imaging of the early-stage changes in the vasculature of tumours that have been treated with photodynamic therapy (PDT). The subjects used were C3H mice with an RIF-1 tumour implanted subcutaneously and allowed to grow for 3 weeks prior to treatment. The experimental groups were PDT-treated $\left(150 \mathrm{~J} / \mathrm{cm}^{2}\right.$ and $\left.50 \mathrm{~J} / \mathrm{cm}^{2}\right)$ and control $\left(150 \mathrm{~J} / \mathrm{cm}^{2}\right.$ light-only and untreated). The laser light exposure was performed at 15 - 30 minutes after the administration of the photosensitizer (BPD-MA). The contrast-enhanced micro-computed tomography imaging procedure consisted of eight-second scans taking place before treatment and up to 24 hours after treatment. The $150 \mathrm{~J} / \mathrm{cm}^{2}$ PDT group showed a significant increase in the ratio of blood volume to tumour volume at 2, 8 and 24 hours after treatment when compared to pre-treatment measurements $(p<$ 0.01). The observed increase in the blood volume to tumour volume at the later time points corresponds to a decrease in epithelial coverage on immunohistochemical stained (CD31) slides for the $150 \mathrm{~J} / \mathrm{cm}^{2}$ PDT group at 24 hours after treatment. This preliminary study indicates that micro-CT can detect compromised vasculature in tumours treated with high-fluence photodynamic therapy as early as $\mathbf{2}$ hours post treatment.
\end{abstract}

Keywords: Micro-Computed Tomography; Contrast Agent; Animal Model; Photodynamic Therapy; Cancer

\section{INTRODUCTION}

Photodynamic therapy (PDT) is a cancer treatment able

"Formerly at Ryerson University. to cause selective destruction of tumours while avoiding damage to adjacent healthy tissue. Depending on the photosensitizer used, the effects induced can be vascular or non-vascular. One photosensitizing agent, benzoporphyrin derivative monoacid ring (BPD-MA), has been shown previously to induce vascular collapse in eye conditions and tumours [1]. The treatment efficacy depends on the time interval between the administration of the photosensitizer and the light exposure as well as the duration of the light delivery. In the 15 to 30 minutes following administration, the photosensitizer remains within the blood vessels, and therefore only vascular effects are obtained upon activation with the laser. Beyond this time interval, the photosensitizer leaks out of the vasculature and into the surrounding tissue allowing for PDT destruction to occur in the surrounding tissue [2-5].

Photosensitizer kinetics and efficacy in the treatment of different tumour types can be studied in preclinical rodent models. For monitoring of the PDT treatment efficacy non-invasively and longitudinally, imaging techniques such as magnetic resonance imaging, ultra- sound, and optical coherence tomography have been investigated [6-9]. In ultrasound, it is difficult to obtain an isotropic representation of the treated tumour as the spatial resolution varies with depth and position of the transducer. In addition, tissue properties change as a result of PDT treatment making registration of images acquired at different time points difficult. Ultrasound is currently being used in cell studies in vitro where depth and tissue inhomogeneity are not a problem [6].

In optical coherence tomography, the resolution exceeds $10 \mu \mathrm{m}$, but the limitation is that only one image at a specific depth can be acquired for each time point. The tissue properties change over time in response to treatment; therefore, in a longitudinal study, it could be difficult to identify and obtain sequential images at the same depth or even in the same plane that was previously cho- 
sen, making the comparison over time difficult [8].

Magnetic resonance imaging (MRI) is a volumetric modality, which eliminates some of the problems associated with ultrasound and optical coherence tomography, such as locating specific planes of the target, and depth penetration. While MRI provides good contrast resolution, the trade-off between scan time and spatial resolution makes MRI a less popular choice [7,9]. As an example, with a resolution of $0.8 \mathrm{~mm}$ at $4.7 \mathrm{~T}$ and a FOV enclosing only the tumour, scan times can be 15 minutes or more [10].

Due to variability in the distribution of the photosensitizer and in the light dose, the best way to follow PDT is by looking at the full volume of the tumour. Micro-CT offers a good compromise in terms of the isotropic representation of the treated volume, short scanning time, and the possibility of performing longitudinal studies. In our study, we used a dynamic micro-CT scanner that provides volumetric images in 8 -second acquisition times. This scanner has been characterized previously [11], and was used for time-course studies of contrast agents [12] to provide snapshots of the contrast enhancement at very precise time points. For obtaining very high temporal-resolution images, the trade-off is in the spatial resolution, with the images acquired at $0.15 \mathrm{~mm}$ isotropic voxel spacing. This resolution is not sufficient to resolve the microvasculature in a tumour, but with the use of contrast agents, the presence of a vessel can be detected.

In this study, we proposed dynamic contrast-enhanced micro-CT for rapid assessment of the vascular effects of PDT treatment as scan times are on the order of seconds, with high-resolution volumetric images and low x-ray dose per scan. The introduction of intravenous contrast agents allows the vasculature to be well visualized in the micro-CT images, improving the contrast between the vessels and underlying soft tissues. One common bloodpool agent in preclinical imaging is Fenestra VC, with an iodine concentration of $50 \mathrm{mg} / \mathrm{mL}$. Previous studies have shown that Fenestra VC gives repeatable enhancement in the blood making it a good match for tumour studies due to its long retention in the vasculature. Ford et al. showed sustained retention of the agent in the blood for 2 - 3 hours [12] after which the contrast agent is collected in the liver. Full accumulation in the liver was observed by Graham et al. at approximately 8 hours after the first Fenestra VC administration and 5 hours after its complete clearance from the blood vessels [13]. Badea et al. successfully tested its application for imaging tumour vasculature [14].

In this study, we monitored the early-stage vascular effects of BPD-MA mediated PDT using contrast-enhanced micro-CT imaging. Images acquired over 24 hours post treatment were analysed quantitatively using image-based measurements of the tumour volume and blood volume. Results from the imaging study were compared with histological analyses.

\section{MATERIALS AND METHODS}

\subsection{Ethics Statement}

All mouse procedures were conducted in accordance with the ethical approval from the Animal Ethics Committee (University Health Network, Toronto, Canada).

\subsection{Animal Model and Tumour Cell Line}

The subjects in this experiment were female $\mathrm{C} 3 \mathrm{H}$ mice (Jackson Laboratories, Bar Harbor, ME, USA) with a mean mass of $23 \pm 2 \mathrm{~g}$. Over the duration of the experiment they were housed in the Animal Resource Centre of the Toronto Medical Discovery Tower (Toronto, Canada). The mice were 11 and 14 weeks old at the time of the cell inoculation and of the treatment, respectively. We shaved off the fur covering the tumour prior to the experimental protocol to avoid attenuation of the laser beam arriving at the tumour.

Radiation-induced fibrosarcoma cells (RIF-1, Ontario Cancer Institute, Toronto, Canada) were cultured in Dulbecco's Modified Eagle's Medium (GIBCO 11900, Rockville USA) with $10 \%$ FBS (Wisent Inc, Quebec, Canada) at $37^{\circ} \mathrm{C}$ and passaged at intervals of $2-3$ days. For inoculation, a pH-buffered solution of RIF-1 cells and Hank's balanced salt solution was prepared to contain $10^{5}$ cells per $0.1 \mathrm{~mL}$ of solution. The solution of suspended cells was delivered subcutaneously into the posterior flank of each mouse. At this location, the cells would develop into a solid tumour over a period of 3 weeks.

\subsection{Study Groups}

The study included 20 mice in total, divided into 4 groups ( 5 animals each) -2 treated and 2 control. The two PDT-treated groups differed by the light fluence used to activate the photosensitizer administered to each treated subject. One group received a fluence of 150 $\mathrm{J} / \mathrm{cm}^{2}$, while the other received $50 \mathrm{~J} / \mathrm{cm}^{2}$. The two control groups were the untreated and the light-only group. The subjects in the light-only group received light with a fluence of $150 \mathrm{~J} / \mathrm{cm}^{2}$ to the surface of the tumour without the prior administration of a photosensitizer. It was not necessary to use a control group to test the effects of the BPD-MA alone as it has been previously shown to be chemically inactive in the absence of light activation [15, $16]$.

\subsubsection{Contrast Agent Administration}

Fenestra VC (ART, Montreal, Canada) containing an iodine concentration of $50 \mathrm{mg} / \mathrm{mL}$ was delivered via tail- 
vein injection at a dose of $0.01 \mathrm{~mL} / \mathrm{g}$ body weight immediately following the pre-contrast scan, and at $15-30 \mathrm{~min}-$ utes before the laser irradiation of the tumour (third timepoint scan). This dose proved to give significant contrast enhancement in previous rodent vascular studies [12]. For scans performed approximately 3 hours or longer after the first contrast agent administration [12], contrast enhancement of the blood was reduced due to clearance of the agent. Hence, for the 8- and 24-hour post-PDT scans, the same dose of contrast agent was re-injected.

\subsubsection{Photodynamic Therapy Treatment}

The photosensitizer (Visudyne, Novartis, Mississauga, Canada) was delivered in a dose of $2 \mathrm{mg} / \mathrm{kg}$ of body weight via tail vein injection. There was a 15 - 30 minute delay between the injection of the photosensitizer and the laser light delivery. This insured localization of the photosensitizer within the blood vessel at the time of its light activation.

The light source was a custom-made $690 \mathrm{~nm}$ diode laser (Princess Margaret Hospital, Toronto, Canada) with a maximum power capacity of $300 \mathrm{~mW}$. The light was delivered to the tissue through a $400 \mu \mathrm{m}$ optical fiber at $100 \mathrm{~mW}$. The laser light exposure was controlled to ensure that the fluence at the tumour surface was uniformly distributed. The exposure time varied from 3 up to 9 minutes. The irradiating beam diameter was matched to the tumour surface area. The range of tumour sizes measured in the mice varied from $4 \times 5 \mathrm{~mm}$ to $5 \times 9 \mathrm{~mm}$ (ellipsoidal shape, measured at the widest points). The total tumour thickness, assessed by caliper measurements and confirmed post mortem, ranged from 4 to $6 \mathrm{~mm}$. The beam exposure time varied from 3 - 9 minutes to reflect the measured changes in tumour volume in each subject.

\subsubsection{Experimental Timeline}

Three weeks after the inoculation of RIF-1 cells, each animal received the combined imaging and treatment protocol. Two micro-CT images were taken prior to treatment: baseline and post-contrast agent scans. After the treatment took place, 8 more scans were performed: 1 immediately following treatment and the others at 0.25 , $0.50,0.75,1,2,8$ and 24 hours after treatment. For each of the 8- and 24-hour scans, Fenestra VC was re-injected prior to imaging.

The mice were kept under inhaled anaesthesia $(1.5 \%$ isofluorane in oxygen) during the combined imaging and treatment session for approximately 1.5 hours (from the baseline scan until the 1-hour post treatment scan), and the respiration, heart rate, and temperature were continuously monitored with a Biovet physiological monitoring device (m2m Imaging Corp., Cleveland, $\mathrm{OH}$, USA). The mice were recovered and subjected to 10 minute anaesthesia intervals for the remaining scans at 2 ,
8 and 24 hours post treatment.

\subsection{Scanning Protocol}

The micro-CT scanner used was the GE Locus Ultra (General Electric Health Care, London, Canada). The 8 -second scans were performed using $80 \mathrm{kVp}$ and $70 \mathrm{~mA}$ tube settings with the mouse resting in a prone position. The protocol consisted of 1000 views per rotation with a 54-by-54 $\mathrm{mm}^{2}$ transaxial field of view. The entrance dose for this image acquisition protocol was previously measured as 0.07 Gy [17]. The image reconstruction algorithm was a modified Feldkamp algorithm for conebeam scanners with $0.15 \mathrm{~mm}$ isotropic voxel spacing.

\subsection{Histology and Immunohistochemistry}

After the final scan, each mouse was euthanized, and the tumours were excised and fixed in buffered formalin for 24 hours prior to staining. Central axial slices were stained with hematoxylin and eosin (H\&E) to visualize the cellular structures within the tumour. One sample in each group also received immunohistochemical staining (CD31), which allows quantifiable assessment of vascular epithelial damage.

Images of immunostained (CD31) and histological (H \&E) slides were taken on a CKX21 microscope with the Olympus IX71 (Olympus Canada Inc., Markham, Canada) using the QCapture Software (QImaging Corp., Surrey, Canada).

\subsection{Image Analysis}

The image analysis was performed using MicroView 2.2 (General Electric Healthcare, London, Canada), a microCT image display and analysis software. In order to quantify vascular changes induced by PDT, we made comparisons among groups and between scan time points. The parameter used for comparison was the ratio of contrast-enhanced blood volume to tumour volume. This ratio shows the progression of the vascular response as a normalized parameter that overcomes the variation in tumour volume between animals.

\subsubsection{Tumour Volume Measurements}

Regions of interest (ROIs) were contoured in the axial plane of the image representing the tumour volume, tumour core, and periphery (both within the previously delineated tumour volume) and the tumour bed (volume of normal tissue adjacent to the tumour). In order to avoid any bias in choosing the tumour boundary location based on contrast enhancement of the blood, we performed the contouring on the image obtained prior to contrast agent administration, and the resulting contours were applied to all images of the same animal. To elimi- 
nate slight movements between the images, all images of the same animal were coordinate-registered to ensure that the ROI was in the correct location. The contours were then interpolated and a three-dimensional rendering of the tumour was generated and the tumour volume was calculated by Microview.

For the tumour volume, the contours were selected to follow the margins of the tumour. To contour the tumour bed, the upper boundary followed the margin of the tumour and extended into the underlying healthy tissue. The lower boundary of the contour followed the same curvature as the upper boundary to produce a bananashaped ROI. Extra care was taken to exclude the femur in all slices to avoid classifying the bone as contrastenhanced blood. Each tumour bed ROI was individually scaled to the size of the tumour.

The contouring of the tumour core was done using the 24 hour post-PDT image. The overall shape of the core was similar to that of the tumour-elliptical-and the diameter of the core was approximately half of the diameter of the tumour. No contouring was needed for the periphery, as it was obtained by image subtraction of the core from the original tumour.

To assess the variability in contouring of the regions, we performed intra-observer and inter-observer studies. For the variability study, only the tumour was contoured from the baseline images of the 5 untreated mice. To assess intra-observer variability, one observer performed the contouring procedure using the same window and level settings three times with approximately one-month interval between contouring sessions. For inter-observer variability, the measurements by the first observer were compared with measurements by two additional observers, who each performed the contouring procedure once using the same window and level settings.

\subsubsection{Blood Volume Measurements}

The image acquired immediately post-contrast injection for the 5 control subjects was used to determine the grey-scale value that would separate the blood from the tumour tissue. An ROI, ranging in size from 1.27 to 1.62 $\mathrm{mm}^{3}$, was drawn such that approximately $50 \%$ of the voxels represented blood, and the rest represented tumour tissue.

Within each ROI, the threshold value separating contrast-enhanced blood voxels and tumour tissue voxels was found [18]. The mean value for the control animals was determined (100 HU) and applied to all images in the study.

For estimates of the blood volume within the tumour, a seeded region-growing algorithm was used to select voxels with a grey-scale value above the threshold (100 $\mathrm{HU})$. The blood volume was obtained within the tumour
ROI, in the core and periphery regions of the tumour, and in the healthy tissue comprising the tumour bed.

\subsubsection{Immunohistochemistry}

For one mouse in each study group, the microscopy images of CD31 stained tissue taken with a $40 \times$ magnification factor were converted to threshold-based binary images using Adobe Photoshop CS3 (Adobe Systems Inc., San Jose, USA). The CD31 stained areas were those of lowest intensity [19-21] — the pixels of grey-scale value of zero after the threshold-based conversion. By summing up all pixels of zero grey-scale value, the total CD31 stained area was obtained, which corresponds to the epithelial coverage.

The ratio of CD31-stained areas over the total tissue area was calculated. This method is an adaptation from a range of other immunohistochemical studies [22-27]. An average over three regions in each tumour section was calculated, and the four groups were compared.

\subsection{Statistical Analysis}

For assessment of the vascular response to treatment, comparisons between treatment groups were made using repeated measures two-way ANOVA with Bonferroni post-hoc tests. The software used for the statistical analysis was Prism 4 (GraphPad Software Inc., San Diego, USA).

\section{RESULTS}

The metric that we report is the blood volume in the region to the volume of the region, where 4 regions were measured: tumour, tumour core, tumour periphery, and healthy tissue in the tumour bed. We compared these 4 measured values over the course of the experiment to show any changes within each treatment group over 24 hours post treatment as compared with the pre-treatment image. We also compared the treatment groups (150 $\mathrm{J} / \mathrm{cm}^{2}$ PDT and $50 \mathrm{~J} / \mathrm{cm}^{2}$ PDT) with the untreated groups $\left(150 \mathrm{~J} / \mathrm{cm}^{2}\right.$ light-only and untreated) to determine whether there were significant differences.

Micro-CT images showing the differences between the tumour vascular response among the groups are given in Figure 1, with multiplanar reformatted images in the axial plane showing the 24-hour post-PDT images of one tumour from each group. The bright regions inside the tumour represent the contrast-enhanced blood, and panel (d) shows increased contrast enhancement in the 150 $\mathrm{J} / \mathrm{cm}^{2}$ PDT tumour compared to the other groups.

\subsection{Blood Volume to Tumour Volume Ratio}

Comparisons between the groups showed a significantly 


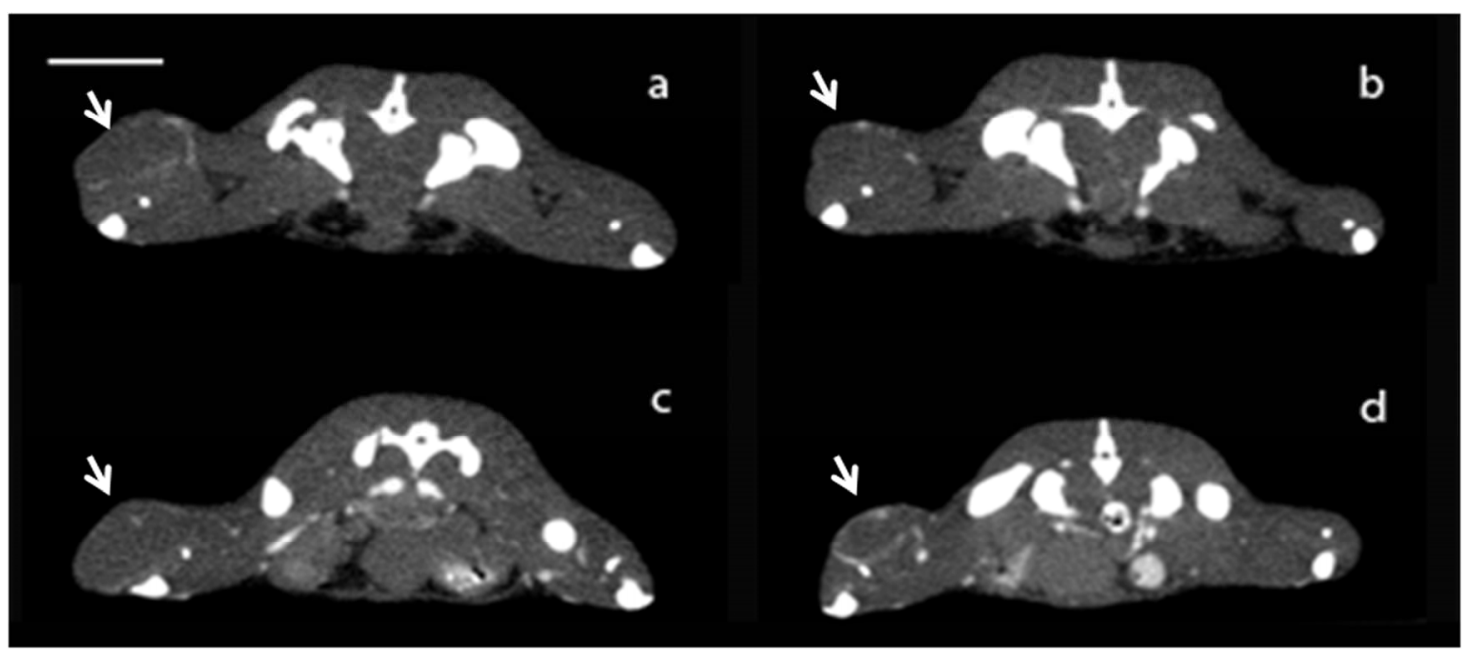

Figure 1. Sample axial view of one mouse in each study group (at the 24-hour post-PDT time-point). (a) Untreated; (b) $150 \mathrm{~J} / \mathrm{cm}^{2}$ light-only; (c) $50 \mathrm{~J} / \mathrm{cm}^{2}$ PDT; and (d) $150 \mathrm{~J} / \mathrm{cm}^{2}$ PDT. Note: all images have an isotropic voxel spacing of $0.15 \mathrm{~mm}$. The scale bar denotes $5 \mathrm{~mm}$, and the arrow points at the location of the tumour.

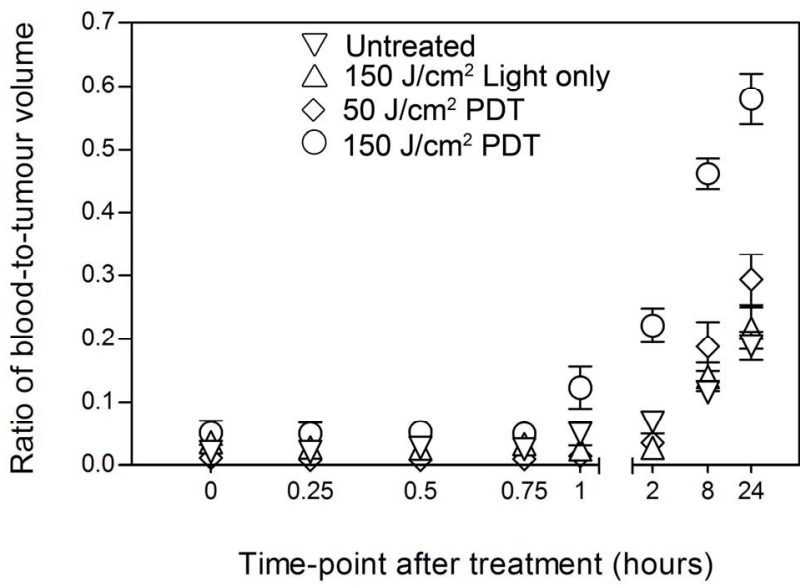

Figure 2. The ratio of blood to tumour volume in the four study groups post-PDT. The timeline includes the time point immediately post-PDT and the 24 -hour post-PDT time point.

higher ratio of blood to tumour volume for the $150 \mathrm{~J} / \mathrm{cm}^{2}$ PDT group compared with the other 3 groups at 2, 8 and 24 hours post treatment $(\mathrm{p}<0.01)$. No significant differences were found at the earlier time points or for the $50 \mathrm{~J} / \mathrm{cm}^{2}$ PDT treatment, $150 \mathrm{~J} / \mathrm{cm}^{2}$ light-only, or control groups. Results are presented in Figure 2 as a mean of the 5 animals in each group for each time point.

The two control groups and the $50 \mathrm{~J} / \mathrm{cm}^{2}$ PDT showed slight blood volume to tumour volume increases at the 8and 24-hour post-PDT time-points when compared to the pre-PDT time-point, which is due to additional injections of the contrast agent at 8 and 24 hours post treatment. Although the contrast enhancement in the blood was reduced at these later time points, the agent had not completely cleared from the blood. It has been shown by Ford et al. [12] that the contrast enhancement of the blood and of the liver parenchyma is equivalent at 8 hours post-injection, indicating that the contrast agent has not completely cleared from the vasculature. As a result of reinjecting the contrast agent, there is a systematic overestimation of the blood volume at 24 hours post treatment for all treated and untreated groups due to incomplete clearance of the contrast agent from the blood at the later time points. Since the same error occurs for all animals, we believe that the trends observed in the study and the conclusions drawn are valid.

\subsection{Tumour Core vs. Tumour Peripheral Changes}

Some vascular effects at the 8- and 24-hour post-PDT time points for the $50 \mathrm{~J} / \mathrm{cm}^{2}$ PDT group may be lost due to averaging effects over the entire tumour. Therefore, separate comparisons in the core and the periphery of the tumour were done to identify any variations in the measurements of blood volume to tumour core or peripheral volume in time or between treatment groups.

In the peripheral region, the ratio of blood to periphery volume results are illustrated in Figure 3(a). In each group, the ratios obtained from the pre-PDT, the 8- and the 24-hour post-PDT scans were compared. In the 150 $\mathrm{J} / \mathrm{cm}^{2}$ PDT group, significant differences were observed at 8 and 24 hours compared with the other 3 groups ( $p<$ 0.05 ). No change in the blood volume-to-periphery volume was detected in the two untreated groups or the 50 $\mathrm{J} / \mathrm{cm}^{2}$ PDT group.

In the core region, the ratio of blood to core volume is illustrated for all 4 groups in Figure 3(b). This ratio was significantly higher for the $150 \mathrm{~J} / \mathrm{cm}^{2}$ PDT group at the 8- and 24-hour post-PDT time-points when compared to the untreated groups $(\mathrm{p}<0.05)$ and for the $50 \mathrm{~J} / \mathrm{cm}^{2}$ PDT group at the 24 hour time-point compared with the un- 

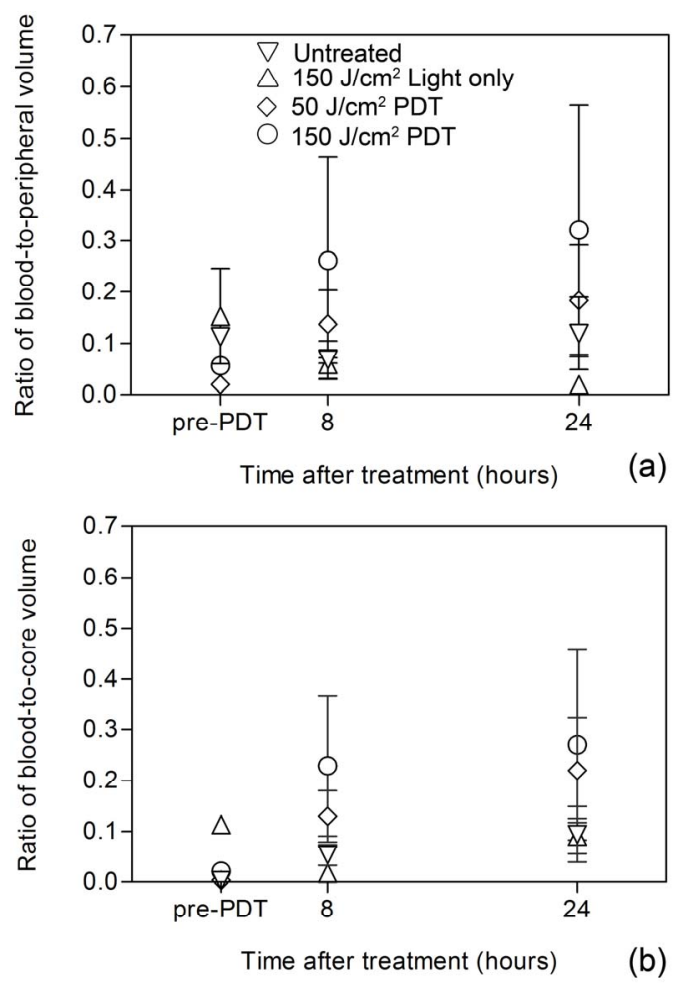

Figure 3. Tumour periphery (a) and core (b). The ratio of the blood to periphery volume (a) and blood-to-core volume is shown at 3 time-points: pre-PDT, and 8 hours and 24 hours post-PDT.

treated groups $(\mathrm{p}<0.05)$. No significant differences were found betwee $n$ the untreated groups $\left(150 \mathrm{~J} / \mathrm{cm}^{2}\right.$ lightonly vs. untreated) or between the treated groups (150 $\mathrm{J} / \mathrm{cm}^{2}$ PDT vs. $50 \mathrm{~J} / \mathrm{cm}^{2}$ PDT).

\subsection{Changes in the Tissue Adjacent to the Tumour}

No blood-volume changes were observed for any of the four groups in the measurements of the healthy tissue in the tumour bed. As expected [4], the vasculature in the tumour bed was not affected by the PDT, suggesting that the treatment successfully targeted the tumour and spared the surrounding healthy tissue.

\subsection{Immunohistochemical Results}

The histology slides, representative of the time point 24 hours after treatment, showed evidence of treatment in the two treated groups (Figure 4). The results obtained from the CD31-based method (Figure 5) showed that the $150 \mathrm{~J} / \mathrm{cm}^{2}$ PDT group had the lowest ratio of epithelial area to total tissue, namely $3 \% \pm 2 \%$.

The standard error in the ratio measurement in each group was $2 \%-5 \%$. Therefore, the $50 \mathrm{~J} / \mathrm{cm}^{2}$ PDT and the light-only groups are indistinguishable at values of $14 \% \pm 3 \%$ and $11 \% \pm 5 \%$, respectively. Lastly, the con-

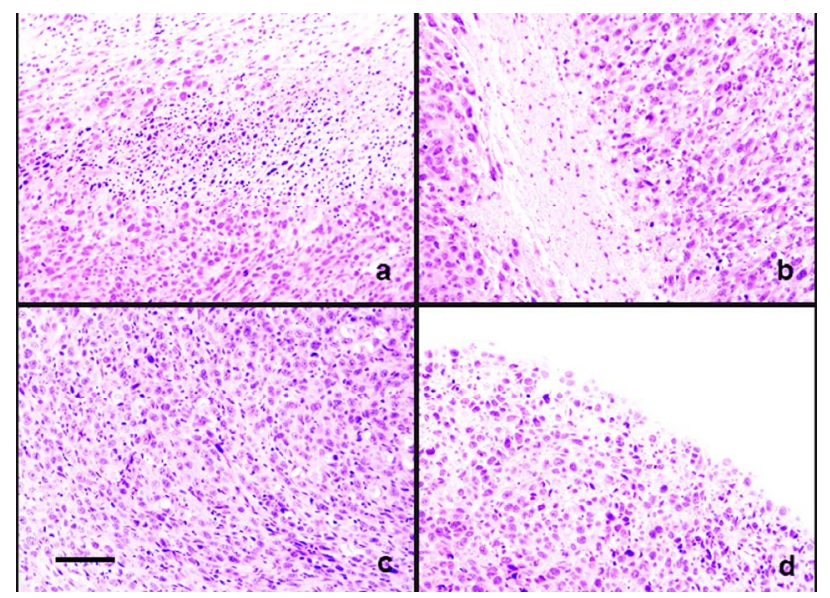

Figure 4. H\&E staining of a tissue sample slice at 24 hours post PDT: (a) $150 \mathrm{~J} / \mathrm{cm}^{2} \mathrm{PDT}$; (b) $50 \mathrm{~J} / \mathrm{cm}^{2} \mathrm{PDT}$; (c) $150 \mathrm{~J} / \mathrm{cm}^{2}$ light-only; and (d) untreated. Note: $20 \times$ magnification factor. The scale bar denotes $100 \mu \mathrm{m}$.

trol group showed the highest ratio of epithelial area to total tissue of $29 \% \pm 5 \%$, as expected given that minimal epidermal damage was induced in this group.

Comparing the untreated tumour to the tumour that received $150 \mathrm{~J} / \mathrm{cm}^{2}$ PDT indicates a reduced amount of epithelium in the tumour vasculature at 24 hours post PDT treatment. The same trend of a decreased amount of epithelium following PDT was also observed immunohistochemically in other studies [19,22,25].

\subsection{Variability of Contouring ROIs}

The tumour volume was contoured on 3 separate occasions by a single observer for the baseline images of the 5 untreated mice. The tumour volume was calculated for each contouring session, and the mean and standard deviation was determined. For the single observer, the standard deviation ranged from $3 \%$ to $8 \%$.

For assessment of interobserver variability, 2 additional observers contoured the same baseline images of the untreated mice using the same window and level settings. The tumour volumes were calculated for each observer. The mean tumour volume and standard deviation were calculated for the 3 observers, with the standard deviation ranging from $10 \%$ to $30 \%$. The means and standard deviations are tabulated for both intra-observer and inter-observer variability in Table 1. As expected, the variability was higher for the inter-observer case. The observers used for the inter-observer case were trained on using the software and were instructed on what to identify as tumour. However, these observers were not as experienced at tumour identification and so they may have selected different tumour margins, especially along the side of the tumour that bordered healthy muscle. To minimize the variability in the measurements, a single experienced observer measured all the values reported in 
this study.

Table 1. Mean and standard deviation of the measurements of the tumour volume as contoured by a single observer on three occasions (intra-observer) and by 3 different observers (interobserver). Images were from the 5 untreated control mice at baseline (no contrast agent present).

\begin{tabular}{ccc}
\hline Untreated subjects & $\begin{array}{c}\text { Intra-observer } \\
\text { volumes }\left(\mathrm{mm}^{3}\right)\end{array}$ & $\begin{array}{c}\text { Inter-observer } \\
\text { volumes }\left(\mathrm{mm}^{3}\right)\end{array}$ \\
\hline Mouse 1 & $160.61 \pm 5.55$ & $139.02 \pm 27.80$ \\
Mouse 2 & $67.45 \pm 5.67$ & $76.05 \pm 10.49$ \\
Mouse 3 & $112.32 \pm 9.04$ & $117.28 \pm 27.53$ \\
Mouse 4 & $55.58 \pm 2.11$ & $45.10 \pm 14.55$ \\
Mouse 5 & $173.79 \pm 5.54$ & $127.12 \pm 26.63$ \\
\hline
\end{tabular}

\section{DISCUSSION}

As expected, the $150 \mathrm{~J} / \mathrm{cm}^{2}$ PDT group showed the strongest vascular response. An increase in the ratio of blood to tumour volume was observed at 2, 8 and 24 hours post treatment. In other studies, different parameters were monitored, including blood flow $[3,5,28,29]$, blood volume [5], and perfusion [30,31] — which were shown to decrease over time (at 3,6 or 24 hours). Our approach of using a ratio of the blood to tumour volume normalizes for the different tumour sizes within the group, which may account for the increased values observed in the treated groups.

Another vascular PDT effect is increased vessel permeability [3,32] due to vessel dilation [5] and destruction of epithelial cells in the vessel walls. A decrease in the

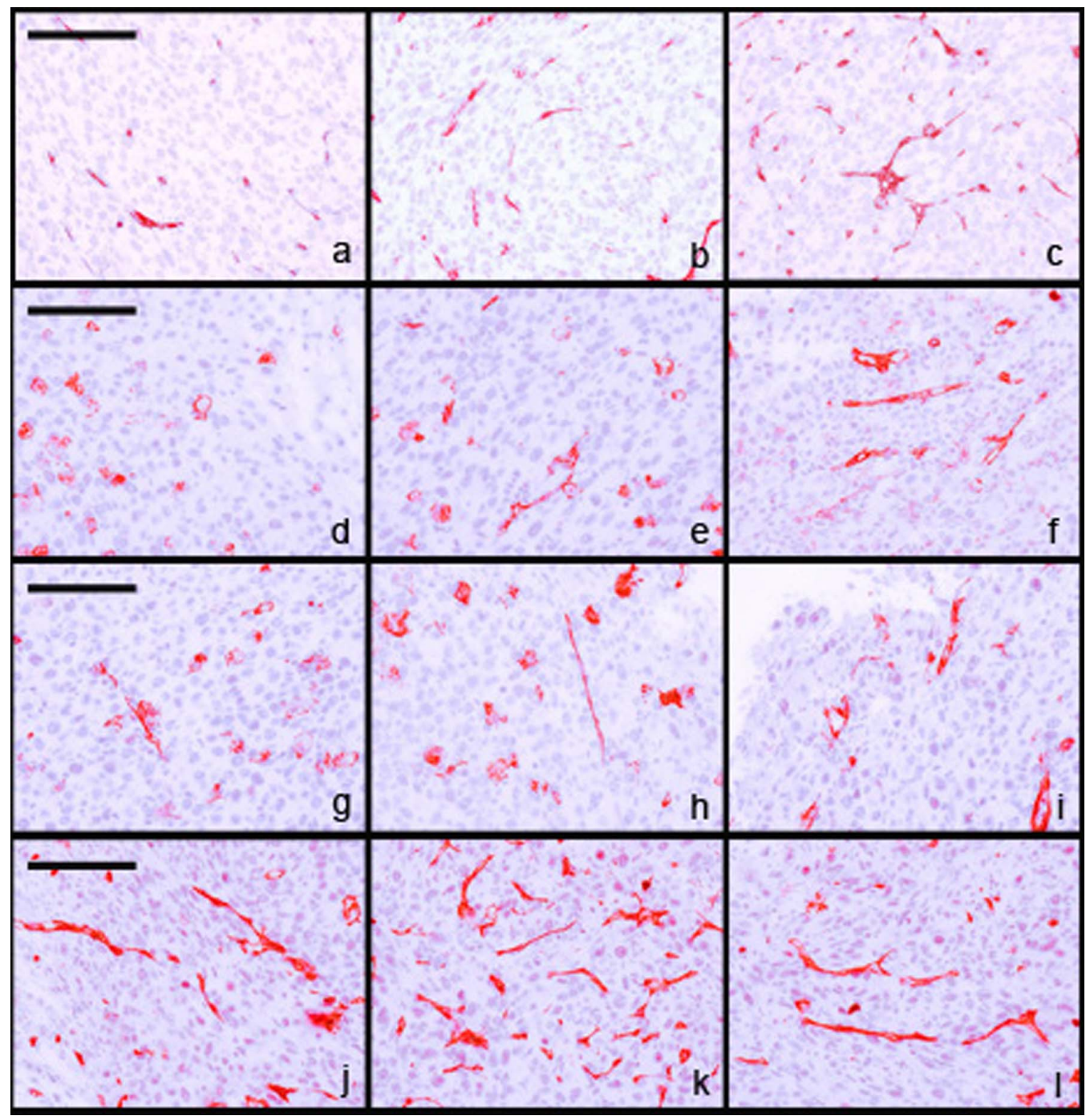

Figure 5. CD31 staining of a tissue sample slice at 24 hours post PDT: (a)-(c) $150 \mathrm{~J} / \mathrm{cm}$ PDT; (d)-(f) 50 $\mathrm{J} / \mathrm{cm}^{2}$ PDT; (g)-(i) $150 \mathrm{~J} / \mathrm{cm}^{2}$ light-only; and (j)-(l) untreated. The images show the small epithelial coverage noticed in the $150 \mathrm{~J} / \mathrm{cm}^{2}$ PDT (a)-(c) when compared to the other three groups. Each row of images represents three locations ( 2 at periphery and 1 at core) on a slide using the $40 \times$ magnification factor. The scale bar denotes $100 \mu \mathrm{m}$. 
vessel epithelial area (vessel damage) in the $150 \mathrm{~J} / \mathrm{cm}^{2}$ PDT group was confirmed by immunohistochemistry (CD31 staining). This stain indicated a smaller ratio of epithelial area to tissue area in the $150 \mathrm{~J} / \mathrm{cm}^{2}$ PDT group at the 24 hour time point when the sample was collected. Therefore, blood (along with contrast agent) could leak out from the tumour vessels into the extravascular space, creating enhancement where no vessel was present. With the same volume of contrast agent re-introduced at 8 and 24 hours after treatment, an accumulation of contrast agent at the site of the tumour will result in an increased measured volume of contrast-enhanced blood.

The $50 \mathrm{~J} / \mathrm{cm}^{2}$ PDT group did not show significantly different results from the control groups for any of the image-based measurements of the blood to tumour ratio or in the periphery of the tumour. A slight increase in the core of the tumour was measured at 24 hours post treatment. The treatment effects of low light-fluence PDT were confirmed histologically. Immunohistochemically, looking at the area of vascular epithelial coverage, the results indicated similarities between the $50 \mathrm{~J} / \mathrm{cm}^{2}$ PDT group and the light-only control groups in the amount of epithelial disruption measured. From these results, we conclude that high-fluence PDT causes increased vascular disruption compared to low fluence PDT and to the untreated groups.

No other imaging studies have separated the vascular effects of PDT into core and peripheral regions of the tumour. The RIF-1 tumour is known to be highly vascularized in both the core and the periphery, with the peripheral area being slightly richer in blood volume than the core [31]. The periphery effects were shown to be observable with contrast-enhanced micro-CT at the later time points ( 8 and 24 hours post PDT) in the high fluence PDT group, with significantly different values compared with all other groups. In the core, differences were observed at 24 hours between the treated and untreated groups, but there were no measurable differences between the $150 \mathrm{~J} / \mathrm{cm}^{2}$ PDT group and the $50 \mathrm{~J} / \mathrm{cm}^{2}$ PDT group. We believe that the PDT may not be as effective in treating the core of the tumour due to attenuation and scattering of the light as it passes through the tissue. Therefore, the core region receives a reduced light fluence compared to the peripheral region. The reduced light fluence in the core may lead to the reduced vascular effects we observed in the micro-CT images, and the inability to distinguish between the two PDT-treated groups. Alternatively, it could be that early necrosis has already begun in the core, making the BPD-MA delivery difficult and thereby minimizing the effects of the photosensitizing light irradiation.

To ensure that the PDT treatment effectively targeted the tumour, and spared the surrounding healthy tissue, blood volume measurements were made in the tumour bed. No change in the blood to tumour bed volume were observed, confirming that the PDT effects did not take place anywhere outside of the tumour [1]. By delivering the light at a suitably low fluence, the observed vascular effects are limited to the tumoural site.

The main limitation of the study is in correlating the experimental end point to give the results biological relevance. In the case of PDT, there is not one single chemical that can be used to confirm or disprove that the PDT treatment took place. Therefore, depending on the aim of the study, different markers can be used that are to be associated with a particular expected effect. Because we applied the laser light within 15 - 30 minutes post-BPD-MA injection, we expected that the photosensitizer would be localized to the vasculature, and therefore the effect of the PDT treatment would be disruption of the vessels within the tumour site. We looked at the vascular epithelial cells with CD31 staining to assess the amount of vascular disruption caused by the BPD-MA mediated PDT treatment. In the CD31 slides, the area covered by the stain demonstrates the effect of PDT on the vascular epithelium only. For assessment of other markers of the treatment of the tumour, additional studies must be performed to follow the animals for a longer period post treatment to determine whether the tumour volume decreases over time, and to provide more histological samples for other types of staining, such as cell proliferation, apoptosis, etc.

A limitation to the image-based method is the manual contouring of the tumour in the micro-CT images. Since the tumour was implanted subcutaneously, the external margins were readily appreciated by the observer. However, if the tumour margin was in contact with the underlying muscle, the inherent contrast in the micro-CT image made separating these two tissue types more difficult. The results of the variability study showed that different observers selected the tumour differently, resulting in a variation in the measured tumour volume. However, the single observer was very consistent in extracting the tumours, which suggests that the data reported, which were measured by a single observer, is internally consistent for our study. The additional precaution of using the same contoured ROI for all images of each mouse ensured that the tumour volume was consistent throughout the study.

Our study has provided a non-invasive means of monitoring the treatment in an in vivo model. In this study, we monitored the tumour vasculature over 24 hours post treatment. From the image-based measurements, we could image as early as 2 hours following 150 $\mathrm{J} / \mathrm{cm}^{2}$ PDT treatment and gain useful information about the vascular disruption that the treatment has induced. Although we acquired a total of 10 micro-CT images, with a total entrance dose to the animal of $0.7 \mathrm{~Gy}$, future 
studies could reduce the number of images to a pretreatment image, and a post treatment image at $2-3$ hours post treatment and obtain statistically significant measurements of the blood to tumour volume, blood to core volume, and blood-to-periphery volume, allowing the effectiveness of the treatment to be assessed within hours. By acquiring the post treatment image within 2 - 3 hours, we would enable imaging with a single contrast injection, which would eliminate a systematic error that we faced in this study, while ensuring stable contrast enhancement in the blood [12].

By identifying the optimal time points for imaging, there are two benefits that could be realized for future studies. First, reducing the number of images to 2 enables the information to be obtained with a 0.14 Gy total dose to the animal. We anticipate that this $\mathrm{x}$-ray dose would be well tolerated by the animal and would not interfere with the tumour growth, as suggested by Foster et al. [17]. Second, the images could be obtained with the use of more standard micro-CT equipment, which would require longer scan times (15 - 40 minutes) but provide higher spatial resolution $(0.05-0.1 \mathrm{~mm})$. Since the contrast enhancement remains stable for up to 4 hours post injection with Fenestra V.C. [12], implementing a higher resolution, long scan at the optimal time points should yield comparable accuracy in measuring the blood volume in the tumour, with the additional ability to visualize some of the smaller vessels within the tumour.

\section{CONCLUSION}

In this study, we used contrast-enhanced micro-CT to monitor BPD-MA mediated photodynamic therapy. Using a blood-pool agent and a dynamic micro-CT scanner, the early time points following treatment were investigated, and measurements of the blood to tumour volume were made from the micro-CT images. Blood-to-tissue ratios were measured regionally within the tumour (periphery and core), and in the surrounding healthy tissue (tumour bed) to demonstrate that the treatment effects were localized to the tumour. The treatment response was strongest in the $150 \mathrm{~J} / \mathrm{cm}^{2}$ PDT group at 2, 8 and 24 hours after the treatment, and was more pronounced in the periphery of the tumour than in the core. Our results indicate that contrast-enhanced micro-computed tomography is a feasible tool for non-invasive monitoring of vascular changes induced by photodynamic therapy. These vascular changes can be detected as early as 2 hours post treatment for $150 \mathrm{~J} / \mathrm{cm}^{2}$ PDT using contrast-enhanced micro-CT.

\section{ACKNOWLEDGEMENTS}

We would like to acknowledge Dr. Brian Wilson at Princess Margaret Hospital in Toronto for sharing his equipment for the photodynamic therapy, Min Rui for her expertise in cell culturing, and Mafe Monroy for performing tail-vein injections and surgical procedures. Funding for this project was from NSERC and Ryerson University.

\section{REFERENCES}

[1] Richter, A.M., Jain, A.K., Canaan, A.J., et al. (1992) Photosensitizing efficiency of two regioisomers of the benzoporphyrin derivative monoacid ring A (BPD-MA). Biochemical Pharmacology, 43, 2349-2358. doi:10.1016/0006-2952(92)90313-8

[2] Ichikawa, K., Takeuchi, Y., Yonezawa, S., et al. (2004) Antiangiogenic photodynamic therapy (PDT) using Visudyne causes effective suppression of tumor growth. Cancer Letters, 205, 39-48. doi:10.1016/j.canlet.2003.10.001

[3] Chen, B., Pogue, B.W., Goodwin, I.A., et al. (2003) Blood flow dynamics after photodynamic therapy with verteporfin in the RIF-1 tumor. Radiation Research, 160, 452-459. doi:10.1667/RR3059

[4] Chen, B., Xu, Y., Roskams, T., et al. (2001) Efficacy of antitumoral photodynamic therapy with hypericin: Relationship between biodistribution and photodynamic effects in the RIF-1 mouse tumor model. International Journal of Cancer, 93, 275-282. doi:10.1002/ijc.1324

[5] Osaki, T., Takagi, S., Hoshino, Y., et al. (2007) Antitumor effects and blood flow dynamics after photodynamic therapy using benzoporphyrin derivative monoacid ring A in KLN205 and LM8 mouse tumor models. Cancer Letters, 248, 47-57. doi:10.1016/j.canlet.2006.05.021

[6] Banihashemi, B., Vlad, R., Debeljevic, B., et al. (2008) Ultrasound imaging of apoptosis in tumor response: Novel preclinical monitoring of photodynamic therapy effects. Cancer Research, 68, 8590-8596. doi:10.1158/0008-5472.CAN-08-0006

[7] Burch, S., London, C., Seguin, B., et al. (2009) Treatment of canine osseous tumors with photodynamic therapy: A pilot study. Clinical Orthopaedics and Related Research, 467, 1028-1034. doi:10.1007/s11999-008-0678-5

[8] Fercher, A.F., Drexler, W., Hitzenberger, C.K., et al. (2003) Optical coherence tomography-Principles and applications. Reports on Progress in Physics, 66, 239-303. doi:10.1088/0034-4885/66/2/204

[9] Celli, J.P., Spring, B.Q., Rizvi, I., et al. (2010) Imaging and photodynamic therapy: Mechanisms, monitoring, and optimization. Chemical Reviews, 110, 2795-2838. doi:10.1021/cr900300p

[10] Gross, S., Gilead, A., Scherz, A., et al. (2003) Monitoring photodynamic therapy of solid tumors online by BOLDcontrast MRI. Nature Medicine, 9, 1327-1331. doi: $10.1038 / \mathrm{nm} 940$

[11] Du, L.Y., Umoh, J., Nikolov, H.N., et al. (2007) A quality assurance phantom for the performance evaluation of volumetric micro-CT systems. Physics in Medicine and Biology, 52, 7087-7108. doi:10.1088/0031-9155/52/23/021

[12] Ford, N.L., Graham, K.C., Groom, A.C., et al. (2006) Time-course characterization of the computed tomogramphy contrast enhancement of an iodinated blood-pool 
contrast agent in mice using a volumetric flat-panel equipped computed tomography scanner. Investigative Radiology, 41, 384-390. doi:10.1097/01.rli.0000197981.66537.48

[13] Graham, K.C., Ford, N.L., MacKenzie, L.T., et al. (2008) Non-invasive quantification of tumour volume in preclinical liver metastasis models using contrast-enhanced $\mathrm{x}$-ray computed tomography. Investigative Radiology, 43, 92-99. doi:10.1097/RLI.0b013e31815603d7

[14] Badea, C.T., Hedlund, L.W., De Lin, M., et al. (2006) Tumor imaging in small animals with a combined micro-CT/micro-DSA system using iodinated conventional and blood pool contrast agents. Contrast Media \& Molecular Imaging, 1, 153-164. doi:10.1002/cmmi.103

[15] Aveline, B., Hasan, T. and Redmond, R.W. (1994) Photophysical and photosensitizing properties of benzoporphyrin derivative monoacid ring A (BPD-MA). Photochemistry and Photobiology, 59, 328-335. doi:10.1111/j.1751-1097.1994.tb05042.x

[16] Henderson, B.W. and Dougherty, T.J. (1992) How does photodynamic therapy work? Photochemistry and Photobiology, 55, 145-157. doi:10.1111/j.1751-1097.1992.tb04222.x

[17] Foster, W.K. and Ford, N.L. (2011) Investigating the effect of longitudinal micro-CT imaging on tumour growth in mice. Physics in Medicine and Biology, 56, 315-326. doi:10.1088/0031-9155/56/2/002

[18] Otsu, N. (1979) A threshold selection method from graylevel histograms. IEEE Transactions on Systems, Man and Cybernetics, 9, 62-66. doi:10.1109/TSMC.1979.4310076

[19] Busch, T.M., Hahn, S.M., Wileyto, E.P., et al. (2004) Hypoxia and Photofrin uptake in the intraperitoneal carcinomatosis and sarcomatosis of photodynamic therapy patients. Clinical Cancer Research, 10, 4630-4638. doi:10.1158/1078-0432.CCR-04-0359

[20] Phongkitkarun, S., Kobayashi, S., Kan, Z., et al. (2004) Quantification of angiogenesis by functional computed tomography in a matrigel model in rats. Academic Radiology, 11, 573-582. doi:10.1016/S1076-6332(03)00728-1

[21] Shaheen, R.M., Davis, D.W., Liu, W., et al. (1999) Antiangiogenic therapy targeting the tyrosine kinase receptor for vascular endothelial growth factor receptor inhibits the growth of colon cancer liver metastasis and induces tumor and endothelial cell apoptosis. Cancer Research, 59, 5412-5416.

[22] Hirakawa, S., Fujii, S., Kajiya, K., et al. (2005) Vascular endothelial growth factor promotes sensitivity to ultraviolet B-induced cutaneous photodamage. Blood, 105, 2392-2399. doi:10.1182/blood-2004-06-2435

[23] Kan, Z., Phongkitkarun, S., Kobayashi, S., et al. (2005)
Functional CT for quantifying tumor perfusion in antiangiogenic therapy in a rat model. Radiology, 237, 151-158. doi:10.1148/radiol.2363041293

[24] McGinley, J.N., Knott, K.K. and Thompson, H.J. (2002) Semi-automated method of quantifying vasculature of 1-methyl-1-nitrosourea-induced rat mammary carcinomas using immunohistochemical detection. Journal of Histochemistry \& Cytochemistry, 50, 213-222. doi: $10.1177 / 002215540205000209$

[25] Ojha, N., Roy, S., He, G., et al. (2008) Assessment of wound-site redox environment and the significance of Rac2 in cutaneous healing. Free Radical Biology and Medicine, 44, 682-691. doi:10.1016/j.freeradbiomed.2007.10.056

[26] Yeh, D.C., Bula, D.V., Miller, J.W., et al. (2004) Expression of leukocyte adhesion molecules in human subfoveal choroidal neovascular membranes treated with and without photodynamic therapy. Investigative Ophthalmology \& Visual Science, 45, 2368-2373. doi:10.1167/iovs.03-0981

[27] Kiessling, F., Krix, M., Heilmann, M., et al. (2003) Comparing dynamic parameters of tumor vascularization in nude mice revealed by magnetic resonance imaging and contrast-enhanced intermittent power Doppler sonogramphy. Investigative Radiology, 38, 516-524. doi:10.1097/01.rli.0000073448.16334.fe

[28] Yu, G., Durduran, T., Zhou, C., et al. (2005) Noninvasive monitoring of murine tumor blood flow during and after photodynamic therapy provides early assessment of therapeutic efficacy. Clinical Cancer Research, 11, 3543-3552. doi:10.1158/1078-0432.CCR-04-2582

[29] Kurohane, K., Tominaga, A., Sato, K., et al. (2001) Photodynamic therapy targeted to tumor-induced angiogenic vessels. Cancer Letters, 167, 49-56. doi:10.1016/S0304-3835(01)00475-X

[30] Chen, B., Roskams, T., Xu, Y., et al. (2002) Photodynamic therapy with hypericin induces vascular damage and apoptosis in the RIF-1 mouse tumor model. International Journal of Cancer, 98, 284-290. doi:10.1002/ijc.10175

[31] Van Geel, I.P., Oppelaar, H., Rijken, P.F., et al. (1996) Vascular perfusion and hypoxic areas in RIF-1 tumours after photodynamic therapy. British Journal of Cancer, 73, 288-293. doi:10.1038/bjc.1996.51

[32] Chen, B., Pogue, B.W., Luna, J.M., et al. (2006) Tumor vascular permeabilization by vascular-targeting photosensitization: Effects, mechanism, and therapeutic implications. Clinical Cancer Research, 12, 917-923. doi:10.1158/1078-0432.CCR-05-1673 